\title{
Salvianolic Acid A Protects Against Oxidative Stress and Apoptosis Induced by Intestinal Ischemia-Reperfusion Injury Through Activation of Nrf2/HO-1 Pathways
}

\author{
Guo Zu ${ }^{\mathrm{a}}$ Tingting Zhou ${ }^{\mathrm{b}} \quad$ Ningwei Che $^{\mathrm{c}}$ Xiangwen Zhang ${ }^{\mathrm{a}}$ \\ aDepartment of Gastroenterology Surgery, The Dalian Municipal Central Hospital Affiliated of Dalian \\ Medical University, Dalian, bepartment of Neurology, The First Affiliated Hospital of Dalian Medical \\ University, Dalian, 'Department of Neurosurgery, The Second Affiliated Hospital of Dalian Medical \\ University, Dalian, China
}

\section{Key Words}

Salvianolic acid A • Intestinal ischemia/reperfusion injury • Oxidative stress •apoptosis • Nrf2/ HO-1 pathways

\begin{abstract}
Background/Aims: Ischemia-reperfusion (I/R) adversely affects the intestinal mucosa. The major mechanisms of I/R are the generation of reactive oxygen species (ROS) and apoptosis. Salvianolic acid A (SalA) is suggested to be an effective antioxidative and antiapoptotic agent in numerous pathological injuries. The present study investigated the protective role of SalA in I/R of the intestine. Methods: Adult male Sprague-Dawley rats were subjected to intestinal I/R injury in vivo. In vitro experiments were performed in IEC- 6 cells subjected to hypoxia/ reoxygenation $(H / R)$ stimulation to simulate intestinal I/R. TNF- $\alpha$, IL-1 $\beta$, and IL- 6 levels were measured using enzyme-linked immunosorbent assay. Malondialdehyde and myeloperoxidase and glutathione peroxidase levels were measured using biochemical analysis. Apoptosis was measured by terminal deoxynucleotidyl transferase mediated dUTP nick-end labeling staining or flow cytometry in vivo and in vitro. The level of reactive oxygen species (ROS) was measured by dichlorodihydrofluorescin diacetate (DCFH-DA) staining. Western blotting was performed to determine the expression of heme oxygenase-1 ( $\mathrm{HO}-1)$, Nrf2 and proteins associated with apoptosis. The mRNA expressions of Nrf2 and HO-1 were detected by quantitative realtime polymerase chain reaction in vivo and in vitro. Results: Malondialdehyde level and myeloperoxidase and glutathione peroxidase, TNF- $\alpha$, IL-1 $\beta$, and IL- 6 levels group in intestinal tissue decreased significantly in the SalA pretreatment groups compared to the I/R group. SalA markedly abolished intestinal injury compared to the I/R group. SalA significantly attenuated apoptosis and increased Nrf2/HO-1 expression in vivo and in vitro. However, Nrf2 siRNA


treatment partially abrogated the above mentioned effects of SalA in H/R-induced ROS and apoptosis in IEC- 6 cells. Conclusion: The present study demonstrated that SalA ameliorated oxidation, inhibited the release of pro-inflammatory cytokines and alleviated apoptosis in I/Rinduced injury and that these protective effects may partially occur via regulation of the Nrf2/ HO-1 pathways.

\section{Introduction}

The small intestinal mucosa acts as a protective barrier from various environmental stimuli, including physical and chemical insults and microbial invasion, and it is likely the most sensitive and vulnerable area to ischemia reperfusion (I/R) injury [1]. The mechanisms of intestinal I/R injury are extensively researched and reviewed in the literature [2]. The production of oxygen free radicals and inflammatory cytokines is one mechanism that leads to intestinal epithelium damage and apoptosis in intestinal I/R injury [3, 4]. Enzymes involved in defense mechanisms that exhibit antioxidant and antiapoptotic activity to protect against free radical-induced damage also play a role [5]. Therefore, elevated levels of antioxidant and antiapoptosis activity may protect against intestinal I/R-induced damage.

Recent data indicated that a key transcription factor for endogenous antioxidative enzymes, NFE2-related factor-2 (Nrf2), plays a central role in the protective effect against oxidative and apoptotic damage [6]. Nrf2 binds to the antioxidant response element (ARE) in the promoter region of several genes, and it is an upstream regulator of the encoding of antioxidative proteins, such as heme oxygenase 1 (HO-1), which antagonize reactive oxygen species (ROS)-induced oxidative stress [7, 8].

Salvianolic acid A (SalA) is a water-soluble component that is extracted from the dried root and rhizome of Salvia miltiorrhiza Bunge (Danshen). SalA exhibits antioxidant, antiinflammatory, and antiplatelet properties. Fig. 1 shows the chemical structure of SalA. Previous studies indicated the beneficial effects of SalA in preventing oxidative stress, platelet aggregation and hepatic cirrhosis [9]. SalA exhibited protective effects in myocardial and cerebral I/R injury $[10,11]$. Salvianolate reduced endotoxin levels, ameliorated injury to the intestinal mucosa, and inhibited the expression of TNF- $\alpha$ and IL- 6 mRNA in the small intestine of cirrhotic rats [12]. However, the effects of SalA on intestinal I/R were not reported. The present study, for the first time, examined the protective effects of SalA on the intestinal mucosa following I/R injury in experimental animals and the role of Nrf2/HO-1 pathway activation.

\section{Materials and Methods}

Animals and experimental procedures

Adult male Sprague-Dawley rats weighing 200-250 $\mathrm{g}$ and raised in an animal house (SPF animal center of Dalian Medical University) were used. The study protocol and experiments were performed according to the guidelines of the Institutional Animal Care and Use Committee of Dalian Medical University, and the Institutional Ethics Committee of Dalian Medical University approved the protocol. The facility is airconditioned, and the temperature was

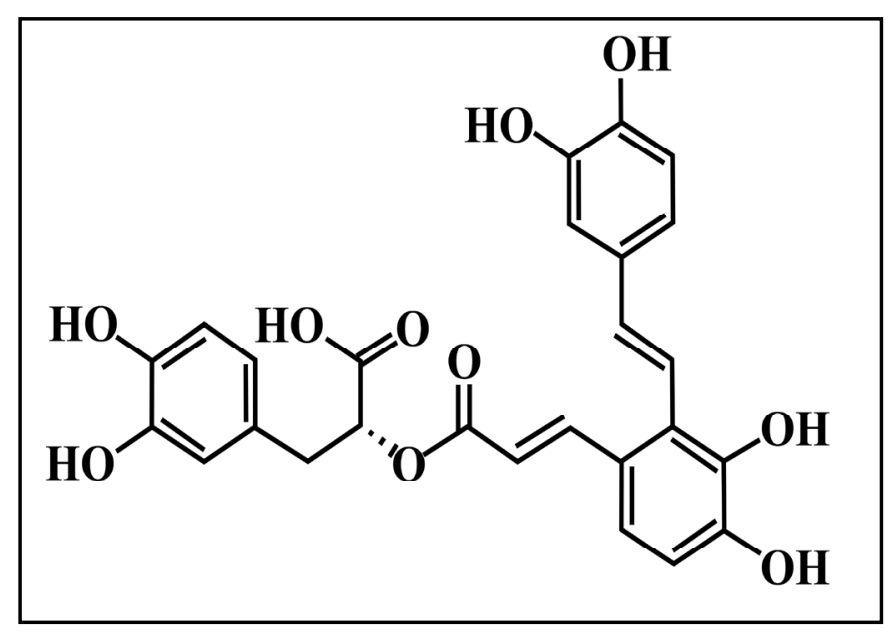

Fig. 1. Chemical structure of SalA. 


\section{Cellular Physiology Cell Physiol Biochem 2018;49:2320-2332

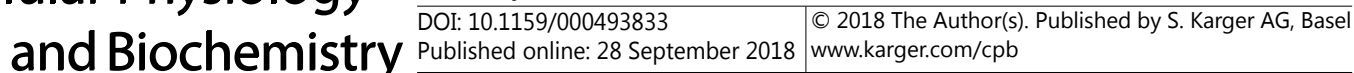 \\ Zu et al.: Salvianolic Acid A Protects Intestinal Ischemia-Reperfusion Injury}

maintained at $21-24^{\circ} \mathrm{C}$, with controlled illumination (12-h light/dark cycle). The rats were fed a standard laboratory diet with water available ad libitum.

The animals were divided into four groups: Control group, I/R Group, SalA(10)+I/R and SalA(20)+I/R (10 animals in each group). Animals in the I/R Group were anesthetized via ether inhalation, and the abdomen was opened using a midline incision. Ischemia to the intestine was induced via clamping of the superior mesenteric artery (SMA) using a microclamp. The abdomen was closed, and the clamp was removed after $60 \mathrm{~min}$ to reperfuse the gut. The abdomen was closed. The abdomen was reopened $6 \mathrm{~h}$ after reperfusion, and the ileum was removed. Ileum tissues were fixed in $10 \%$ formalin. The abdominal cavity was opened in control group, but the bowels and SMA were not clamped. The abdomen was closed, and 2 inches of ileum specimens were collected at the end of the experiments. SalA $10 \mathrm{mg} / \mathrm{kg}$ and $20 \mathrm{mg} / \mathrm{kg}$ were administered daily for 3 days via gavage in the SalA(10)+I/R and SalA(20)+I/R groups, respectively, followed by I/R as described above.

\section{Histopathological analysis}

Fixed ilea from each experimental group were embedded in paraffin blocks. The blocks were cut into 4- $\mu \mathrm{m}$ thick sections and stained with hematoxylin and eosin. Sections were examined using a $10 \times$ objective. Injury to the intestinal mucosa was quantified in five sections from each rat and scored using the Chiu score [13].

Measurement of intestinal interleukin-6, tumor necrosis factor- $\alpha$ and interleukin-1 $\beta$ using enzyme-linked immunosorbent assay

Rats were sacrificed, and a segment (300 mg) of the terminal ileum $10 \mathrm{~cm}$ proximal to the ileocecal valve was immediately frozen in liquid nitrogen. The intestinal tissues were homogenized using a tissue homogenizer and centrifuged at $12,000 \mathrm{rpm}$ at $4{ }^{\circ} \mathrm{C}$ for $15 \mathrm{~min}$. The supernatant was collected for ELISA. TNF- $\alpha$, IL-1 $\beta$, and IL-6 in intestines were measured using commercial ELISA kits (Bio Swamp Life Science Inc., Wuhan, China) in accordance with the manufacturer's protocols.

\section{Biochemical analysis of intestinal tissues}

The in vivo intestinal tissues of rats were homogenized on ice in normal saline, and the supernatant was collected. Malondialdehyde (MDA) level and myeloperoxidase (MPO) and glutathione peroxidase activities in the supernatants were measured spectrophotometrically according to the manufacturer's protocols (Nanjing Jiancheng Corp., Nanjing, China). The results were calculated as nmol/g protein, U/g protein, and $\mathrm{U} / \mathrm{g}$ protein, respectively.

\section{Western blot analyses}

Intestinal tissues or cells were lysed in RIPA buffer (Beyotime, Jiangsu, China). The protein concentration in each sample was determined using a BCA protein assay kit (Bio-Rad, CA, USA). Protein samples $(20 \mu \mathrm{g})$ were separated on $12 \%$ SDS-PAGE gels and transferred onto polyvinylidenedifluoride (PVDF) membranes (Bio-Rad Laboratories, Hercules, CA, USA). The membranes were blocked with 5\% nonfat dry milk in Trisbuffered saline containing $0.1 \%$ Tween 20 and incubated with specific antibodies against Nrf2 (1:200), HO-1 (1:200), caspase-3 (1:200), Bax (1:400), Bcl-2 (1:400), and $\beta$-actin (1:1000) (Santa Cruz, CA, USA) overnight at $4{ }^{\circ} \mathrm{C}$. Membranes were incubated with anti-rabbit IgG or anti-mouse IgG secondary antibodies (1:2000; ZSGB-Bio, Beijing, China) for $2 \mathrm{~h}$ at $37^{\circ} \mathrm{C}$. Proteins were visualized using ECL reagents (Beyotime, Jiangsu, China). The expression of $\beta$-actin was used as an internal control.

\section{Total RNA extraction and $q R T-P C R$}

Intestinal tissues (in vivo) of rats and IEC-6 cells (in vivo) were collected, and total RNA was extracted using TRIzol (Takara, Dalian, China) according to the manufacturer's instructions. The quantity and purity of the obtained total RNA samples were determined using UV spectroscopy (NanoDrop 2000 Spectrophotometer, Thermo Fisher Scientific, Waltham, MA, USA). cDNA was synthesized using the TransScript All-in-One First-Strand cDNA Synthesis SuperMix for qPCR Kit (TransGen Biotech, Beijing, China). Polymerase chain reaction (PCR) was performed. The following PCR primers were used: Rat Nrf2 


\section{Cellular Physiology Cell Physiol Biochem 2018;49:2320-2332

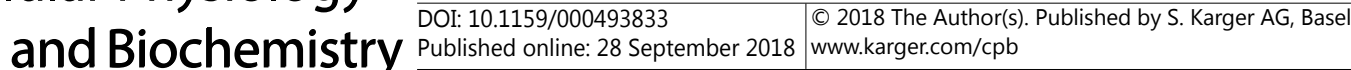 \\ Zu et al.: Salvianolic Acid A Protects Intestinal Ischemia-Reperfusion Injury}

(sense): 5'-CACAGTGCTCCTATGCGTGA-3', antisense: 5'-TTCTGGGCGGCGACTTTAT T-3'; HO-1 (sense): 5'-GGTGATGGCCTCCTTGTACC-3', antisense: 5'-GTGG GGCATAGACTGGGTTC-3'; and $\beta$-actin (sense): 5'-GGAAATCGTGCGTGACAT TAAAG-3', antisense: 5'-CGGCAGTGGCCATCTCTT-3'. Real-time PCR analyses were performed to detect mRNA expression using a TransStart Top Green qPCR SuperMix kit (TransGen Biotech, Beijing, China) according to the manufacturer's instructions. Samples were run using a 7500 Fast Real-time PCR System (Applied Biosystems). The following reverse transcription conditions were used: 42 ${ }^{\circ} \mathrm{C}$ for $15 \mathrm{~min}$, followed by $5 \mathrm{~s}$ at $85{ }^{\circ} \mathrm{C}$ for RT/RI inactivation. qPCR was performed using the following conditions: $30 \mathrm{~s}$ at $94{ }^{\circ} \mathrm{C}$ for denaturation; $5 \mathrm{~s}$ at $94{ }^{\circ} \mathrm{C}$ for annealing, and $30 \mathrm{~s}$ at $60^{\circ} \mathrm{C}$ for extension. $\beta$-actin was used as a housekeeping gene.

\section{Detection of epithelial apoptosis}

Tissues were fixed in $4 \%$ paraformaldehyde, and $10-\mu \mathrm{m}$ frozen sections were prepared. Intestinal epithelial apoptosis was investigated using terminal deoxynucleotidyl transferase mediated dUTP nickend labeling (TUNEL) staining and an in situ cell death detection POD kit (Roche, Penzberg, Germany) in accordance with the manufacturer's instructions. The slides were rinsed with phosphate-buffered saline (PBS) for $10 \mathrm{~min}$ at room temperature. Slides were incubated with $0.1 \%$ Triton X-100 for $8 \mathrm{~min}$, rinsed with PBS for $15 \mathrm{~min}$ and $50 \mu \mathrm{L}$ of the TUNEL reaction mixture was added and incubated for $60 \mathrm{~min}$ at 37 ${ }^{\circ} \mathrm{C}$. Slides were rinsed three times with PBS and examined under a fluorescence microscope. The ratio of TUNEL-positive cells was calculated as follows: (number of apoptotic cells/total number counted) $\times 100 \%$. Each assay was performed in a blinded manner, and the experiment was repeated three times.

\section{Cell culture and $H / R$ model}

We used rat intestinal jejunal crypt cells (IEC-6) exposed to H/R as a model, which is a common model of intestinal I/R injury. IEC-6 cells were cultured in Dulbecco's modified Eagle's medium supplemented with $10 \%$ fetal bovine serum (Gibco, Grand Island, NY) in a humidified atmosphere of air with $5 \% \mathrm{CO}_{2}$ at $37^{\circ} \mathrm{C}$ Cellular $\mathrm{H} / \mathrm{R}$ conditions were created via incubation in a microaerophilic system (Thermo Scientific, Waltham, MA) at $5 \% \mathrm{CO}_{2}$ and $1 \% \mathrm{O}_{2}$ balanced with $94 \% \mathrm{~N}_{2}$ for $6 \mathrm{~h}$ to simulate intestinal I/R in vivo. The cells were cultured in normoxic conditions $\left(95 \%\right.$ air and $5 \% \mathrm{CO}_{2}$ at $\left.37^{\circ} \mathrm{C}\right)$ for $6 \mathrm{~h}$ of reoxygenation as previously described [14]. IEC- 6 cells were pre-treated or not with SalA, at concentrations ranging from 5 to $20 \mu \mathrm{g} / \mathrm{ml}$, for variable periods of time (3-24h) and then subjected to H/R.

\section{CCK-8 assay for cytotoxicity}

Cell viability and survival were determined using the tetrazolium salt Cell Counting Kit-8 (CCK-8, Dojindo Molecular Technologies, Inc. Tokyo, Japan) according to the manufacturer's recommendations. Briefly, $2 \times 10^{3}$ cells were plated in 96-well microtiter plates and treated with different SalA concentrations for $6 \mathrm{~h}$. Cells were washed with $100 \mu \mathrm{L}$ DMEM at various time points after treatment, and $10 \mu \mathrm{L}$ of CCK-8 was added. The wells were incubated for $2 \mathrm{~h}$ in normoxic conditions. Absorbance at $450 \mathrm{~nm}$ was measured using an enzyme-linked immunosorbent assay microplate reader (Thermo, Vantaa, Finland). Cell viability was defined relative to an untreated control.

\section{Small interfering RNA transfection}

IEC- 6 cells were plated on 6 -well plates at a density of $1 \times 10^{5}$ cells per dish. Cells for Nrf2 small interfering RNA (siRNA) transfection were transfected with siRNA complexed with Lipofectamine 2000 (Invitrogen, Carlsbad, CA), according to manufacturer's instructions, at 50-60\% confluency. Cells were changed to fresh culture medium after $6 \mathrm{~h}$, and protein expression was examined using Western blot analysis. The siRNA of Nrf2 was purchased from Genepharma (Shanghai, China).

\section{Measurement of cell apoptosis}

Cell apoptosis was assessed using flow cytometry and an Annexin V-FITC/PI Apoptosis Detection Kit from Invitrogen, according to the manufacturer's instruction. The percentage of cell apoptosis was determined using Cell Quest Pro software. 


\section{Cellular Physiology Cell Physiol Biochem 2018;49:2320-2332 and Biochemistry \begin{tabular}{l|l} 
DOI: 10.1159/000493833 & (c) 2018 The Author(s). Published by S. Karger AG, Basel \\
www.karger.com/cpb
\end{tabular}

\section{Measurement of intracellular ROS assay}

In vitro IEC-6 cell ROS production was detected using the fluorescent probe dichlorodihydrofluorescin diacetate (DCFH-DA) staining from Molecular Probes (Invitrogen) according to the protocols described in our previous study [15].

\section{Statistical analysis}

All data are expressed as the means \pm standard deviation (SD) of at least 3 independent experiments. Statistical significance was determined using a nonparametric, 2-tailed Mann-Whitney U test for data with a non-normal distribution. Parametric unpaired two-tailed Student's tests (to compare two groups) or one-way ANOVA (for more than two groups) was used for data with a normal distribution, and the multiple comparisons between variables were carried out the using "Bonferonni post hoc" test. If the variables were not in conformance with the normal distribution, Kruskal-Wallis test was carried out and the Mann Whitney $\mathrm{U}$ test was utilized for the comparison of variables and "Bonferonni" correction test for the significance of statistical data. All analyses were performed using SPSS software (version 19.0; SPSS Inc, Chicago, IL). Graphs were plotted using Prism 5.0 (GraphPad Software, San Diego, CA). A value of $\mathrm{P}<0.05$ was chosen to indicate statistical significance.

\section{Results}

\section{Histopathological studies}

Injury to the intestinal mucosa was quantified using the Chiu score. (Fig. 2). Histopathological analyses of control animals (Fig. 2A) revealed a normal mucosal pattern. Group I/R animals exhibited severe mucosal damage, including villous edema, vascular congestion, and hemorrhage, compared to the sham group. However, the intestinal mucosa was preserved in group SalA(10) + I/R and SalA(20) + I/R animals, and the injury was much less severe compared to the I/R animals. The difference in Chiu score was significantly better in the $\mathrm{SalA}(10)+\mathrm{I} / \mathrm{R}$ and SalA(20) $+\mathrm{I} / \mathrm{R}$ groups than the I/R group $(P<0.05)$ (Fig. 2B).

Tissue malondialdehyde, myeloperoxidase and glutathione-peroxidase

The levels of small intestinal MDA and GPx are good indicators of lipid peroxidation and ischemic damage. MPO activity is an inflammatory marker of leukocyte infiltration. Intestinal tissue MDA, MPO and GPx levels in the I/R group increased significantly compared

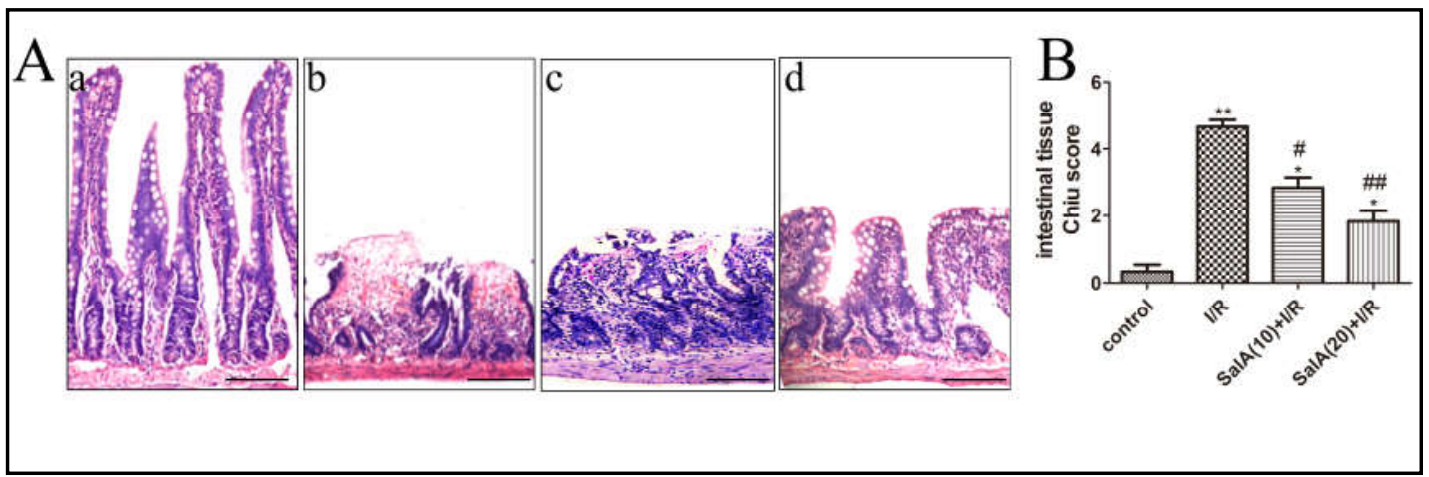

Fig. 2. Histopathological changes in the intestinal mucosa of control, $I / R, S a l A(10)+I / R$ and SalA(20)+I/R groups. (A) Tissue sections of small intestinal mucosa stained with H\&E in different groups. a, Control group; b, I/R group; c, SalA(10)+I/R; d, SalA(20)+I/R. (original magnification $\times 200$ ). (B) Chiu scores of the intestinal mucosa of the control, I/R, SalA(10)+I/R and SalA(20)+I/R groups. The results are representative of five separate experiments; data are the means \pm SD. H\&E, hematoxylin and eosin; I/R, ischemia/reperfusion. ${ }^{*} \mathrm{P}<0.05$ compared to the control group; ${ }^{* *} \mathrm{P}<0.01$ compared to the control group; ${ }^{\#} \mathrm{P}<0.05$ compared to the I/R group; ${ }^{\# \#} \mathrm{P}<0.01$ compared to the I/R group. 


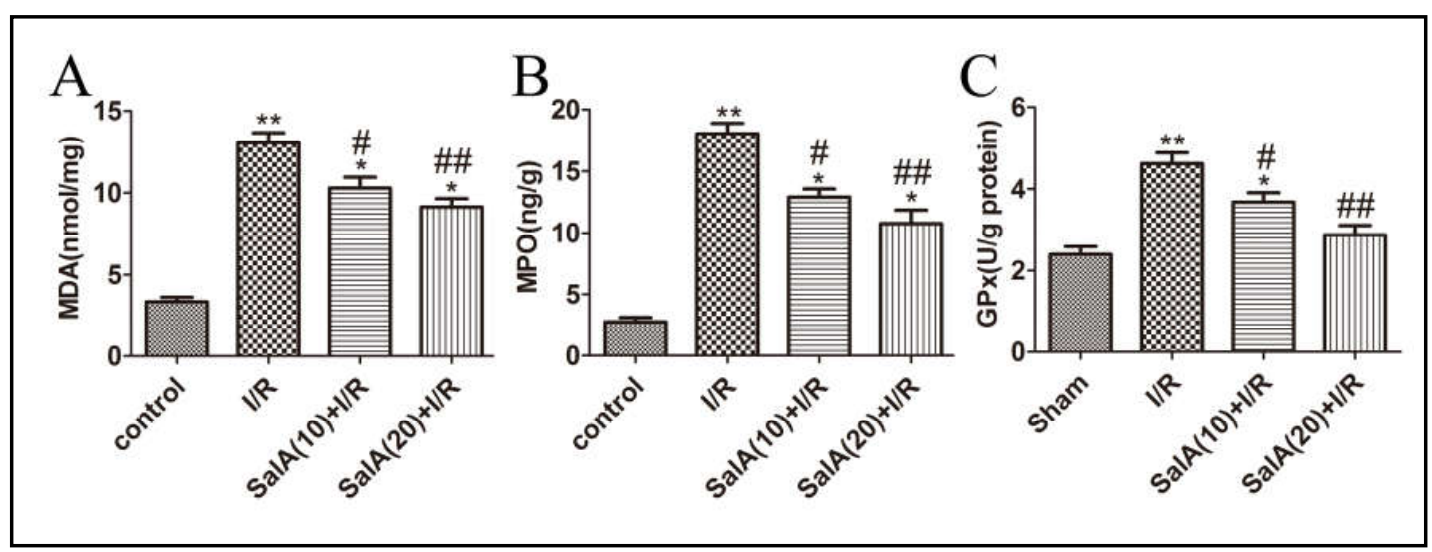

Fig. 3. Effects of SalA on tissue MDA, MPO and GPx in the ileum after I/R. MDA, MPO and GPx levels in the intestinal tissue were elevated significantly after $6 \mathrm{~h}$ of reperfusion in the I/R groups compared to sham rats, and SalA significantly reduced MDA, MPO and GPx levels (A-C, respectively). The results are representative of five separate experiments; data are the means $\pm \mathrm{SD}$. ${ }^{*} \mathrm{P}<0.05$ compared to the control group; ${ }^{* *} \mathrm{P}<0.01$ compared to the control group; ${ }^{\#} \mathrm{P}<0.05$ compared to the I/R group; ${ }^{\#} \mathrm{P}<0.01$ compared to the I/R group.

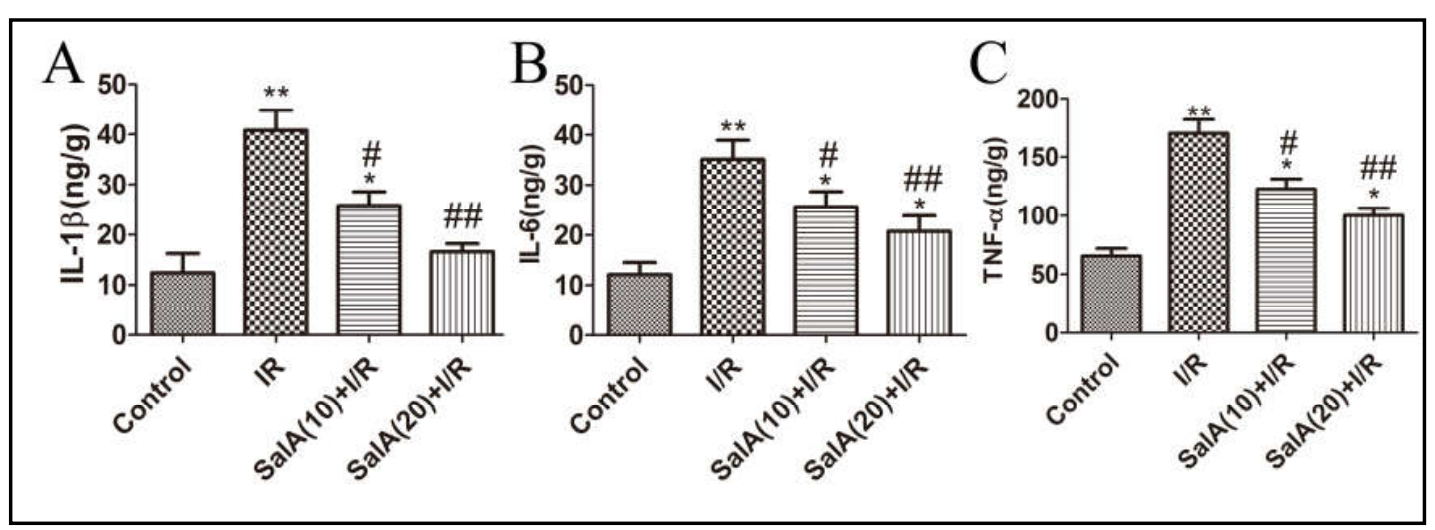

Fig. 4. Effects of SalA on cytokine levels in the ileum after I/R. TNF- $\alpha$, IL-1 $\beta$, and IL-6 levels in intestinal tissue were elevated significantly after $6 \mathrm{~h}$ of reperfusion in the I/R groups compared to sham rats (A-C, respectively), and SalA significantly reduced TNF- $\alpha$, IL-1 $\beta$, and IL- 6 levels (A-C, respectively). The results are representative of five separate experiments; data are the means $\pm \mathrm{SD}$. ${ }^{*} \mathrm{P}<0.05$ compared to the control group; ${ }^{* *} \mathrm{P}<0.01$ compared to the control group; ${ }^{\#} \mathrm{P}<0.05$ compared to the I/R group; ${ }^{\# \#} \mathrm{P}<0.01$ compared to the I/R group.

to the control group (Fig. 3). SalA markedly decreased intestinal tissue MDA, MPO and GPx to levels that were comparable with the I/R group.

\section{Tissue cytokine levels}

TNF- $\alpha$, IL-1 $\beta$, and IL- 6 levels in the intestinal tissue of the I/R group increased significantly compared to the control group, and SalA treatment abrogated these increases (Fig. 4). SalA obviously decreased TNF- $\alpha$, IL- 6 and IL-1 $\beta$ levels in intestinal tissue compared to the I/R group.

Intestinal tissue caspase-3, Bax, and Bcl-2 protein and TUNEL

TUNEL staining evaluated the antiapoptotic effect of SalA (Fig. 5A). I/R significantly increased the number of TUNEL-positive cells in rat intestines compared to the Control group $(P<0.05)$. SalA treatment reduced the number of TUNEL-positive cells in the intestines of rat subjected to I/R $(P<0.05)$. 


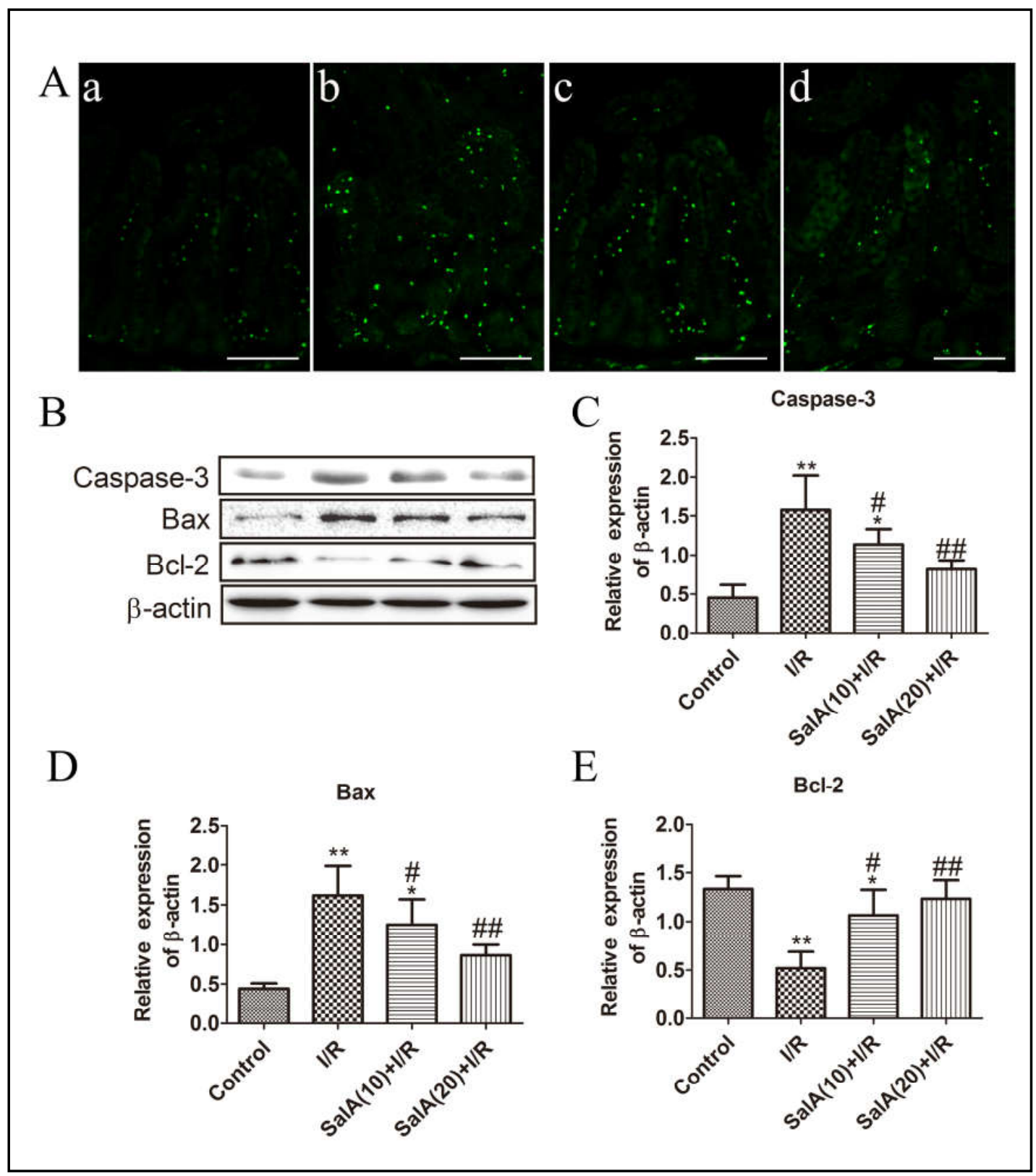

Fig. 5. TUNEL-positive cells and protein expression of caspase-3, Bax, Bcl-2 in rat intestinal tissue. (A) TUNEL-positive cells were observed in intestinal epithelial cells. a, Control group; b, I/R group; c, SalA(10)+I/R; d, SalA(20)+I/R (original magnification $\times 200$ ). (B-E) Protein expression of caspase-3, Bax and Bcl-2 were analyzed using Western blot. The results are representative of three separate experiments; data are the means $\pm \mathrm{SD}$. ${ }^{*} \mathrm{P}<0.05$ compared to the control group; ${ }^{* *} \mathrm{P}<0.01$ compared to the control group; ${ }^{\#} \mathrm{P}<0.05$ compared to the I/R group; ${ }^{\# \#} \mathrm{P}<0.01$ compared to the I/R group.

Western blot analysis revealed that caspase- 3 and Bax protein expression in the I/R group were higher compared to the control group $(\mathrm{P}<0.01)$, and $\mathrm{Bcl}-2$ protein expression in the I/R group was lower than the control group (Fig. 5B-E). SalA attenuated the increase in caspase- 3 and Bax protein expression compared to the I/R group $(\mathrm{P}<0.05)$. SalA significantly increased Bcl-2 protein expression compared to the I/R group $(\mathrm{P}<0.05)$. 
Fig. 6. Quantitative qPCR (A) and Western blot (B) analysis of Nrf2/ HO-1 mRNA expression. Significantly lower Nrf2/ HO-1 mRNA expression was observed in the I/R group. Nrf2/HO-1 expression were lower in the $\operatorname{SalA}(10)+\mathrm{I} / \mathrm{R}$ and $\quad \operatorname{SalA}(20)+\mathrm{I} / \mathrm{R}$ groups compared to the control group, and it was stronger compared to the I/R

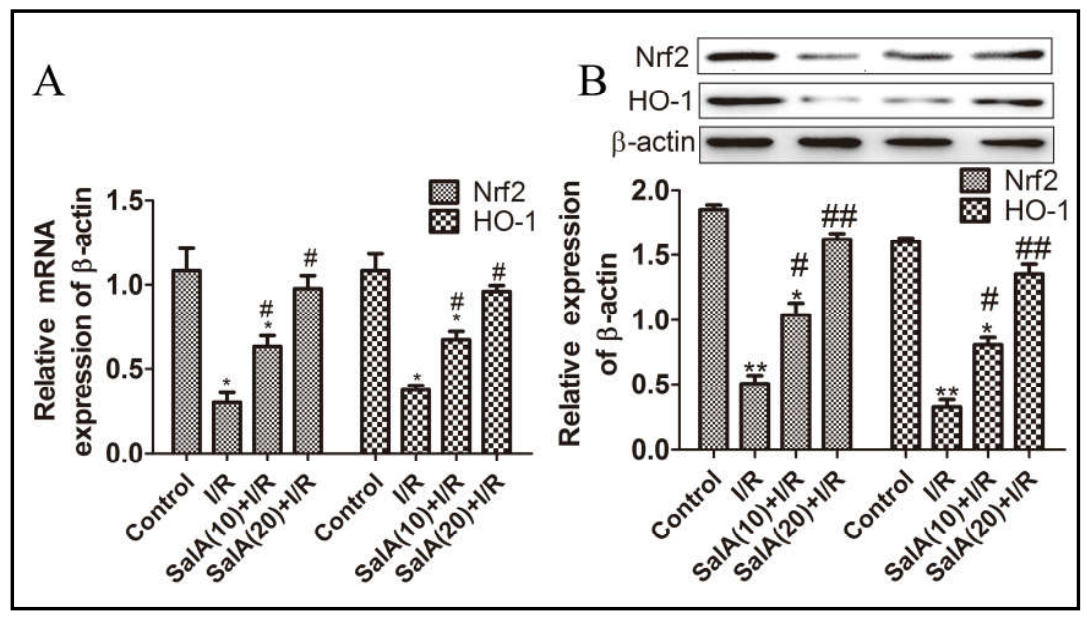
group. The results are representative of three separate experiments; data are the means $\pm \mathrm{SD}$. ${ }^{*} \mathrm{P}<0.05$ compared to the control group; ${ }^{* *} \mathrm{P}<0.01$ compared to the control group; ${ }^{\#} \mathrm{P}<0.05$ compared to the I/R group; ${ }^{\# \# ~} \mathrm{P}<0.01$ compared to the I/R group.

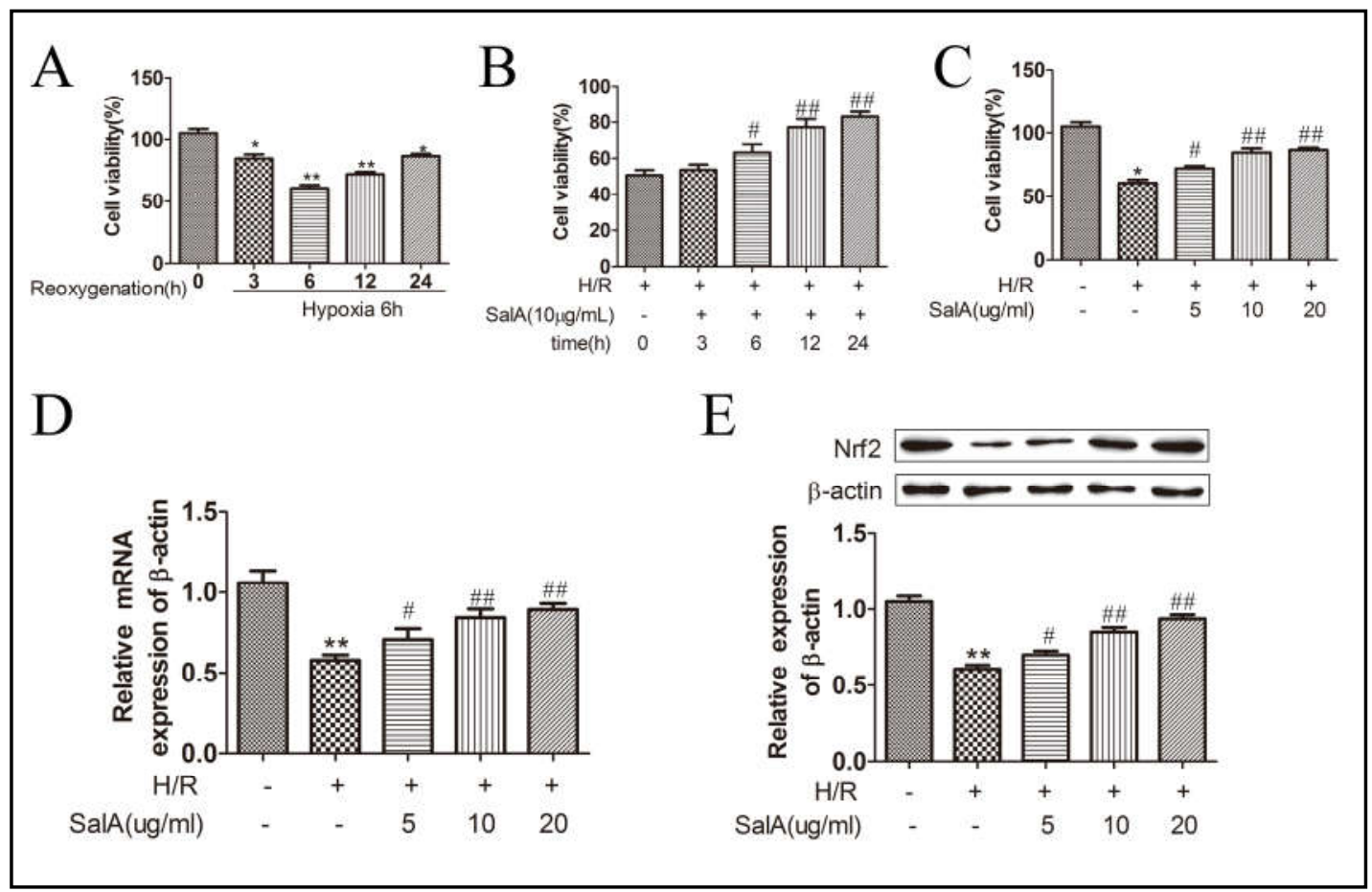

Fig. 7. SalA promotes survival of IEC- 6 cells under H/R conditions in vitro. (A) IEC- 6 cells were exposed to hypoxic environment for $6 \mathrm{~h}$ and reoxygenated for $3,6,12$, or $24 \mathrm{~h}$. The viable cells were determined using the CCK-8 assay. (B) IEC-6 cells were exposed to H/R and pretreated with SalA(10 $\mu \mathrm{g} / \mathrm{ml})$ for 3, 6, 12 , or $24 \mathrm{~h}$. Viable cells were determined using the CCK-8 assay. (C) IEC-6 cells were pretreated with SalA $(5,10$, and $20 \mu \mathrm{g} / \mathrm{mL})$ for $6 \mathrm{~h}$, exposed to hypoxia $6 \mathrm{~h}$ followed by reoxygenation for $6 \mathrm{~h}$. Viable cells were determined using the CCK-8 assay. The results are representative of eight separate experiments; data are the means \pm SD. (D-E) Representative mRNA and protein expression levels of Nrf2 in IEC- 6 cells pretreated with SalA $(5,10$, and $20 \mu \mathrm{g} / \mathrm{mL})$ for $6 \mathrm{~h}$, exposed to hypoxia $6 \mathrm{~h}$ followed by reoxygenation for $6 \mathrm{~h}$. The results are representative of three separate experiments; data are the means $\pm \mathrm{SD}$. ${ }^{*} \mathrm{P}<0.05$ versus control group; ${ }^{* *} \mathrm{P}<0.01$ versus control group; ${ }^{\#} \mathrm{P}<0.05$ versus $\mathrm{H} / \mathrm{R}$ group; ${ }^{\#} \mathrm{P}<0.01$ versus $\mathrm{H} / \mathrm{R}$ group. 


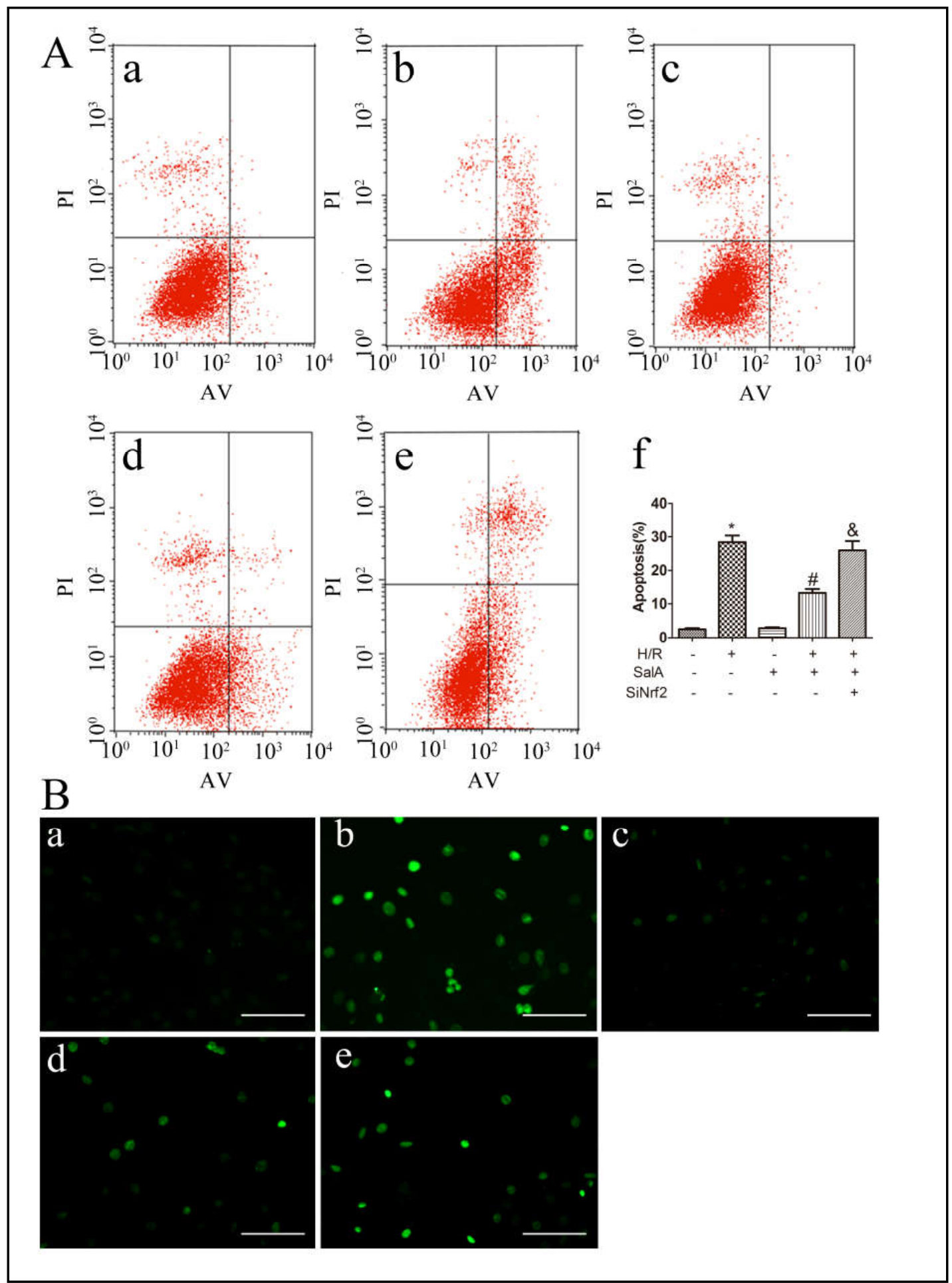

Fig. 8. Nrf2 activation protects IEC-6 cells against H/R-induced apoptosis and oxidative stress. (A) Apoptosis detection of IEC- 6 cells using flow cytometry in different groups (a, Control; b, H/R; c, Control+SalA; d, H) $\mathrm{R}+\mathrm{SalA}$; e, $\mathrm{H} / \mathrm{R}+\mathrm{SalA}+\mathrm{SiNrf2} \mathrm{f}$, the results are representative of three separate apoptosis detection using flow cytometry; data are the means \pm SD.). (B) ROS determination in each group (a, Control; b, H/R; c, Control+SalA; d, H/R+SalA; e, H/R+SalA+SiNrf2). All data were presented as the means $\pm S D, n=3 .{ }^{*} \mathrm{P}<0.05$ versus control group; ${ }^{\mathrm{P}}<0.05$ versus $\mathrm{H} / \mathrm{R}$ group; ${ }^{\mathrm{P}}<0.05$ versus $\mathrm{H} / \mathrm{R}+\mathrm{SalA}$ group. 


\section{Cellular Physiology Cell Physiol Biochem 2018;49:2320-2332

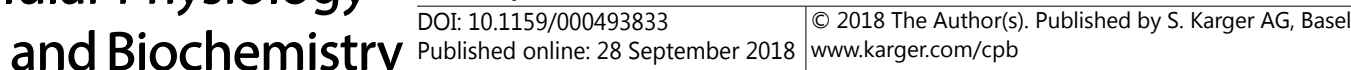 Zu et al.: Salvianolic Acid A Protects Intestinal Ischemia-Reperfusion Injury}

\section{Expression levels in the $\mathrm{Nrf2/HO-1}$ pathway}

The I/R group exhibited lower expression levels of Nrf2/HO-1 on qPCR and Western blots compared to the control group (Fig. 6). Nrf2/HO-1 expression on qPCR and Western blots were higher in the SalA(10)+I/R and SalA(20)+I/R groups compared to the I/R group. There was no obvious difference in Nrf2/HO-1 expression between the SalA(10)+I/R and SalA(20)+I/R groups.

\section{SalA-induced Nrf2 expression under $H / R$ conditions in vitro}

An H/R model of IEC- 6 cells was established to further investigate the mechanisms underlying the protective effects of SalA. SalA pretreatment effectively suppressed H/Rtriggered apoptosis in a time- and concentration-dependent manner (Fig. 7B and 7C). SalA administration induced the Nrf2 mRNA and protein expression in IEC-6 cells in a concentration-dependent manner (Fig. 7D and 7E), which is consistent with the in vivo results.

\section{Protective effects of SalA against H/R-induced apoptosis and ROS in vitro}

IEC- 6 cells were treated with SalA only or SalA and Nrf2 siRNA for $6 \mathrm{~h}$ prior to exposure to $\mathrm{H} / \mathrm{R}$ to examine whether Nrf2 mediated the antioxidant and antiapoptotic activities of SalA. Increased cellular apoptosis and ROS were observed after H/R. SalA pretreatment diminished H/R-induced apoptosis and ROS in IEC- 6 cells. However, the antioxidant and antiapoptotic effects of SalA were abrogated in the presence of Nrf2 siRNA (Fig. 8). These results indicate that Nrf2 mediates the protective effects of SalA against H/R-induced oxidative stress and apoptosis.

\section{Discussion}

The deleterious effect of reperfusion on the intestinal mucosa after ischemia is the result of multiple complex factors that damage the mucosa. ROS and apoptosis are the major mechanisms of I/R injury. The use of antioxidants and antiapoptosis agents decreased the effects of reperfusion on the intestinal mucosa $[5,16]$.

SalA is a water-soluble phenolic acid extracted from the dried root and rhizome of Salvia miltiorrhiza, which is commonly called "Danshen" in China [17]. Modern medical research describes the many beneficial effects of SalA. SalA scavenges lipid free radicals and inhibits lipid peroxidation [18]. Notably, SalA exhibits a variety of pharmacological functions, including anti-inflammation, antioxidant, and antiplatelet effects. However, little is known about the function of SalA in protecting intestinal epithelium cells from I/R injury-induced apoptosis and ROS.

Previous findings reported that MDA and GPx levels increased significantly after intestinal I/R, which indicates that lipid peroxidation and inflammatory markers of leukocyte infiltration play important roles in the pathogenesis of I/R injury [19-21]. Pro-inflammatory factors, such as TNF- $\alpha$, IL- $1 \beta$, and IL- 6 , are potent activators of neutrophils and induce tissue damage. These factors are significant contributors to the increased microvascular permeability and perivascular and interstitial edema in I/R injury [22]. Our study measured intestinal tissue MDA, MPO and GPx levels as indexes of oxidative burst and inflammatory markers of leukocyte infiltration and TNF- $\alpha$, IL-1 $\beta$, and IL- 6 as pro-inflammatory molecules. We demonstrated that SalA ameliorated the acute inflammatory response and systemic oxidative stress of I/R-induced intestinal injuries.

Apoptosis is implicated in numerous physiological and pathological conditions, including intestinal I/R. Numerous regulatory molecules control this process, which mediated by apoptotic signaling. The ratio of Bcl-2/Bax protein determines cell survival or death after I/R $[23,24]$. The cleavage of caspase- 3 is the final step of the process that initiates the apoptotic signaling pathway. The results of the present study demonstrated that SalA attenuated the 


\section{Cellular Physiology Cell Physiol Biochem 2018;49:2320-2332

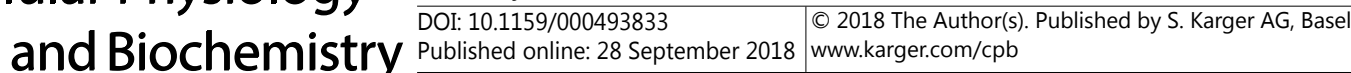 \\ Zu et al.: Salvianolic Acid A Protects Intestinal Ischemia-Reperfusion Injury}

increase in caspase-3 protein expression and Bcl-2/Bax ratio compared to the I/R group. These results indicate that SalA exerts its intestinal protective effect via the upregulation of Bcl-2 protein expression and downregulation of Bax and caspase-3 protein expression, which inhibit apoptosis in the intestinal epithelium.

Nrf2 adjusts regularly evolving cellular procedures, such as migration, growth, and apoptosis [25]. Nrf2 is a crucial modulator of HO-1 expression [26]. Nrf2/HO-1 signaling is a vital path in the apoptosis process [27]. HO-1 is a stress-responsive enzyme that produces antioxidants, anti-inflammatory agents, and regulators of apoptosis [28]. HO-1 is upregulated and protects cells from apoptosis in the rat myocardium in response to I/R injury [29]. Liu et al. found that apoptosis negatively correlated with Nrf2 and HO-1 expression, which ultimately affected the expression of cleaved caspase-3 [30]. A previous study indicated that reduced activity of the Nrf2/HO-1 pathway significantly promoted cytoprotection in the intestine [31]. SalA was an effective Nrf2/HO-1 pathway activator in our study. SalA exhibited potent antioxidant and antiapoptotic properties via the promotion of Nrf2/HO-1 pathway expression by down-regulating cytokines and inflammatory and oxidative factors. These protective effects of SalA support SalA as a novel Nrf2/HO-1 pathway agent to combat intestinal I/R injury.

\section{Conclusion}

In conclusion, the present study demonstrated that SalA ameliorated ROS, proinflammatory cytokine production and apoptosis in I/R-induced intestinal injury. SalA may provide a novel prophylactic strategy for the treatment of I/R-induced intestinal injury in vivo and in vitro, which partially occurred via activation of the $\mathrm{Nrf} 2 / \mathrm{HO}-1$ signaling pathway in vitro. SalA is a promising therapy for I/R-induced intestinal injury with striking efficacy and safety, but it requires further investigation.

\section{Acknowledgements}

This work was supported by grants from the National Natural Science Foundation of China (No. 81700465).

\section{Disclosure Statement}

The authors declare no conflicts of interest.

\section{References}

1 Guan Y, Worrell RT, Pritts TA, Montrose MH: Intestinal ischemia-reperfusion injury: reversible and irreversible damage imaged in vivo. Am J Physiol Gastrointest Liver Physiol 2009;297:G187-196.

-2 Dong M H, Kaunitz J D: Gastroduodenal mucosal defense. Curr Opin Gastroenterol 2006;22:599-606.

3 Bhattacharyya A, Chattopadhyay R, Mitra S, Crowe SE: Oxidative stress: an essential factor in the pathogenesis of gastrointestinal mucosal diseases. Physiol Rev 2014;94:329-354.

-4 Grootjans J, Hodin CM, de Haan JJ, Derikx JP, Rouschop KM, Verheyen FK, van Dam RM, Dejong CH, Buurman WA, Lenaerts K: Level of activation of the unfolded protein response correlates with Paneth cell apoptosis in human small intestine exposed to ischemia/reperfusion. Gastroenterology 2011;140:529-539.

5 Liu KX, Li C, Li YS, Yuan BL, Xu M, Xia Z, Huang WQ: Proteomic analysis of intestinal ischemia/reperfusion injury and ischemic preconditioning in rats reveals the protective role of aldose reductase. Proteomics 2010;10:4463-4475. 


\section{Cellular Physiology Cell Physiol Biochem 2018;49:2320-2332

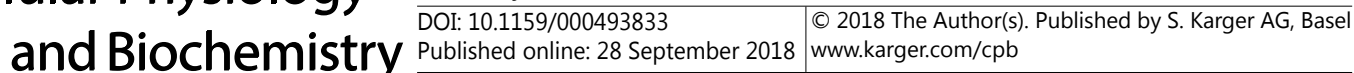

6 Taguchi K, Motohashi H, Yamamoto M: Molecular mechanisms of the Keap1-Nrf2 pathway in stress response and cancer evolution. Genes Cells 2011;16:123-140.

7 Han J, Wang M, Jing X, Shi H, Ren M, Lou H: (-)-Epigallocatechin gallate protects against cerebral ischemiainduced oxidative stress via Nrf2/ARE signaling. Neurochem Res 2014;39:1292-1299.

8 Li L, Dong H, Song E, Xu X, Liu L, Song Y: Nrf2/ARE pathway activation, HO-1 and NQO1 induction by polychlorinated biphenyl quinone is associated with reactive oxygen species and PI3K/AKT signaling. Chem Biol Interact 2014;209:56-67.

-9 Ho JH, Hong CY: Salvianolic acids: Small compounds with multiple mechanisms for cardiovascular protection. J Biomed Sci 2011;18:30.

10 Pan H, Li D, Fang F, Chen D, Qi L, Zhang R, Xu T, Sun H: Salvianolic acid A demonstrates cardioprotective effects in rat hearts and cardiomyocytes after ischemia/reperfusion injury. J Cardiovasc Pharmacol 2011;58:535-542.

11 Jiang M, Wang XY, Zhou WY, Li J, Wang J, Guo LP: Cerebral protection of salvianolic acid A by the inhibition of granulocyte adherence.Am J Chin Med 2011;39:111-120.

12 Yang DH, Ye ZY, Jin B, He XJ, Zhang Q, Zhou WM, Xu WJ, Lu HX: Salvianolate inhibits cytokine gene expression in small intestine of cirrhotic rats. World J Gastroenterol 2011;17:1903-1909.

13 Guneli E, Cavdar Z, Islekel H, Sarioglu S, Erbayraktar S, Kiray M, Sokmen S, Yilmaz O, GokmenN: Erythropoietin protects the intestine against ischemia/ reperfusion injury in rats. Mol Med 2007;13:509517.

14 Zu G, Yao J, Ji A, Ning S, Luo F, Li Z, Feng D, Rui Y, Li Y, Wang G, Tian X: Nurr1 promotes intestinal regeneration after ischemia/reperfusion injury by inhibiting the expression of p21 (Waf1/Cip1). J Mol Med (Berl) 2017;95:83-95.

15 Zu G, Guo J, Che N, Zhou T, Zhang X: Protective effects of ginsenoside Rg1 on intestinal ischemia/ reperfusion injury-induced oxidative stress and apoptosis via activation of the Wnt/ $\beta$-catenin pathway. Sci Rep 2016;6:38480.

-16 Liu SH, Ma K, Xu XR, Xu B: A single dose of carbon monoxide intraperitoneal administration protects rat intestine from injury induced by lipopolysaccharide. Cell Stress Chaperones 2010;15:717-727.

17 Wang L, Zhou GB, Liu P, Song JH, Liang Y, Yan XJ, Xu F, Wang BS, Mao JH, Shen ZX, Chen SJ, Chen Z: Dissection of mechanisms of Chinese medicinal formula realgar-indigo naturalis as an effective treatment for promyelocytic leukemia. Proc Natl Acad Sci USA 2008;105:4826-4831.

18 Guo Y, Xu X, Li Q, Li Z, Du F: Anti-inflammation effects of picroside 2 in cerebral ischemic injury rats. Behav Brain Funct 2010;6:43.

19 Liu KX, Li YS, Huang WQ, Chen SQ, Wang ZX, Liu JX, Xia Z: Immediate postconditioning during reperfusion attenuates intestinal injury. Intensive Care Med 2009;35:933-942.

20 Grootjans J, Hundscheid IH, Lenaerts K, Boonen B, Renes IB, Verheyen FK, Dejong CH, von Meyenfeldt MF, Beets GL, Buurman WA: Ischaemia-induced mucus barrier loss and bacterial penetration are rapidly counteracted by increased goblet cell secretory activity in human and rat colon. Gut 2013;62:250-258.

21 Xu H, Li Y, Che X, Tian H, Fan H, Liu K: Metabolism of salvianolic acid A and antioxidant activities of its methylated metabolites. Drug Metab Dispos 2014;42:274-281.

-22 Bertoni S, Arcaro V, Vivo V, Rapalli A, Tognolini M, Cantoni AM, Saccani F, Flammini L, Domenichini G, Ballabeni V, Barocelli E: Suppression of inflammatory events associated to intestinal ischemia-reperfusion by 5-HT1A blockade in mice. Pharmacol Res 2014;81:17-25.

-23 Lee SJ, Jung YH, Oh SY, Song EJ, Choi SH, Han HJ: Vibrio vulnificusVvhA induces NF- $\kappa B-d e p e n d e n t$ mitochondrial cell death via lipid raft-mediated ROS production in intestinal epithelial cells. Cell Death Dis 2015;6:1655.

24 Wang W, Sun L, Xiao W, Yang H: Essential role of angiotensin receptors in the modulation of intestinal epithelial cell apoptosis. J Pediatr Gastroenterol Nutr 2013;57:562-569.

25 Li P, Su L, Li X, Di W, Zhang X, Zhang C, He T, Zhu X, Zhang Y, Li Y: Remote limb ischemic postconditioning protects mouse brain against cerebral ischemia/reperfusion injury via upregulating expression of Nrf2, HO-1 and NQO-1 in mice. Int J Neurosci 2015;17:1-8.

-26 Sun GY, Li R, Cui J, Hannink M, Gu Z, Fritsche KL, Lubahn DB, Simonyi A: Withania somnifera and its with anolides attenuate oxidative and inflammatory responses and up-regulate antioxidant responses in BV-2 microglial cells. NeuroMolecular Med 2016;18:241-252. 


\section{Cellular Physiology Cell Physiol Biochem 2018;49:2320-2332

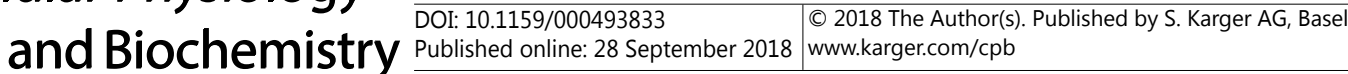 \\ Zu et al.: Salvianolic Acid A Protects Intestinal Ischemia-Reperfusion Injury}

27 Lei X, Lei L, Zhang Z, Cheng Y: Neuroprotective effects of lycopene pretreatment on transient global cerebral ischemia-reperfusion in rats: the role of the Nrf2/HO-1 signaling pathway. Mol Med Rep 2016;13:412-418.

28 Fan J, Xu G, Jiang T, Qin Y: Pharmacologic induction of Heme Oxygenase-1 plays a protective role in diabetic retinopathy in Rats Heme Oxygenase-1 in diabetic retinopathy in rats. Invest Ophthalmol Vis Sci 2012;53:6541-6556.

29 Sun MH, Pang JH, Chen SL, Kuo PC, Chen KJ, Kao LY, Wu JY, Lin KK, Tsao YP: Photoreceptor protection against light damage by AAV-mediated overexpression of heme oxygenase-1. Invest Ophthalmol Vis Sci 2007;48:5699-5707.

30 Liu C, Vojnovic D, Kochevar IE, Jurkunas UV: UV-A irradiation activates Nrf2-regulated antioxidant defense and induces p53/Caspase3-dependent apoptosis in corneal endothelial Cells UV-A activates Nrf2 and induces p53 in corneal endothelial cells. Invest Ophthalmol Vis Sci 2016;57:2319-2327.

-31 Liu C, Zhu C, Wang G, Xu R, Zhu Y: Higenamine regulates Nrf2-HO-1-Hmgb1 axis and attenuates intestinal ischemia-reperfusion injury in mice. Inflamm Res 2015;64:395-403. 